\title{
Postdischarge Recovery after Acute Pediatric Lung Disease Can Be Quantified with Digital Biomarkers
}

\author{
Matthijs D. Kruizingaa, b, c Allison Molla,b Ahnjili Zhuparris ${ }^{a} \quad$ Dimitrios Ziagkos $^{a}$ \\ Frederik E. Stuurman $^{\mathrm{a}, \mathrm{c}}$ Marianne Nuijsink ${ }^{\mathrm{b}}$ Adam F. Cohen $^{\mathrm{a}, \mathrm{c}}$ \\ Gertjan J.A. Driessen ${ }^{b, d}$ \\ ${ }^{a}$ Centre for Human Drug Research, Leiden, The Netherlands; bJuliana Children's Hospital, HAGA Teaching Hospital, \\ The Hague, The Netherlands; ' Leiden University Medical Centre, Leiden, The Netherlands; ${ }^{\mathrm{d}}$ Maastricht University \\ Medical Centre, Leiden, The Netherlands
}

\section{Keywords}

Pediatrics - Wearable $\cdot$ Acute lung disease $\cdot$ At-home recovery $\cdot$ Linear mixed model $\cdot$ Pneumonia $\cdot$ Wheezing . Asthma

\begin{abstract}
Background: Pediatric patients admitted for acute lung disease are treated and monitored in the hospital, after which full recovery is achieved at home. Many studies report in-hospital recovery, but little is known regarding the time to full recovery after hospital discharge. Technological innovations have led to increased interest in home-monitoring and digital biomarkers. The aim of this study was to describe at-home recovery of 3 common pediatric respiratory diseases using a questionnaire and wearable device. Methods: In this study, patients admitted due to pneumonia ( $n=30$ ), preschool wheezing $(n=30)$, and asthma exacerbation (AE; $n=11)$ were included. Patients were monitored with a smartwatch and a questionnaire during admission, with a 14-day recovery period and a 10-day "healthy" period. Median compliance was calculated, and a mixed-effects model was fitted for physical activity and heart rate (HR) to describe the recovery period, and the physical activity recovery trajectory was correlated to
\end{abstract}

karger@karger.com www.karger.com/res

Karger $\stackrel{\text { ' }}{5}$
(C) 2021 The Author(s)

Published by S. Karger AG, Basel

This is an Open Access article licensed under the Creative Commons Attribution-NonCommercial-4.0 International License (CC BY-NC) (http://www.karger.com/Services/OpenAccessLicense), applicable to the online version of the article only. Usage and distribution for commercial purposes requires written permission. respiratory symptom scores. Results: Median compliance was $47 \%$ (interquartile range [IQR] 33-81\%) during the entire study period, 68\% (IQR 54-91\%) during the recovery period, and $28 \%$ (IQR 0-74\%) during the healthy period. Patients with pneumonia reached normal physical activity 12 days postdischarge, while subjects with wheezing and AE reached this level after 5 and 6 days, respectively. Estimated mean physical activity was closely correlated with the estimated mean symptom score. HR measured by the smartwatch showed a similar recovery trajectory for subjects with wheezing and asthma, but not for subjects with pneumonia. Conclusions: The digital biomarkers, physical activity, and HR obtained via smartwatch show promise for quantifying postdischarge recovery in a noninvasive manner, which can be useful in pediatric clinical trials and clinical care.

(c) 2021 The Author(s)

Published by S. Karger AG, Basel

\section{Introduction}

Pediatric patients who are admitted to the pediatric ward for acute lung disease are treated as in-patients until the clinical condition is stable enough for safe discharge. Although the clinical condition is improved 
at discharge, complete recovery is usually achieved at home.

While much is known about in-hospital recovery, little data are available regarding the time to full recovery after hospital discharge. Studies researching acute lung disease, such as community-acquired pneumonia (CAP) and preschool wheezing (PW) define recovery time as the duration of hospital stay, but these studies do not describe the at-home recovery period once patients have been discharged [1-3]. One study investigated at-home recovery time in children admitted for asthma exacerbation (AE), but this definition relied on spirometry only, which does not always correspond with symptoms in children $[4,5]$. As a result, this important clinical disease characteristic does not reach clinical reviews or reference texts, while insights into the duration to full recovery could be valuable for patients, their parents, clinicians, and clinical researchers $[6,7]$. It would allow for better insights in expected shortterm disease burden and also for investigating the effect of treatments, such as steroids and antibiotics, beyond the hospital setting $[8,9]$.

Current methods to follow subjects in a home-setting, such as questionnaires, have limitations such as recall bias and are inherently subjective [10]. Frequent monitoring at a subject's home by a physician is time-consuming and expensive, and daily hospital visits place an unwanted burden on both the child and parent [11, 12]. Technological innovations have led to wearables and other devices that can continuously measure health parameters such as physical activity, heart rate (HR), and sleep pattern. When combined with electronic patient-reported outcomes, remote monitoring platforms can collect both objective and subjective high-resolution data in an athome setting, which decreases the burden for children significantly and may even allow for a personalized medicine approach $[13,14]$.

However, before implementing digital biomarkers in clinical care or clinical trials, extensive fit-for-purpose technical and clinical validation in the target population is necessary. Technical validation consists of investigating whether a device actually captures what is claimed on a technical level, whereas clinical validation focuses on the tolerability, difference between patients and controls, and correlation with traditional biomarkers [15]. One of the final clinical validation criteria in a recently published validation strategy is the ability to detect clinically meaningful change after a health event such as a hospital admission, and this study aimed to address that criterion for several candidate digital biomarkers [15]. The aim of this pilot study was to investigate the postadmission recovery time of children admitted due to pneumonia, PW, and asthma and to evaluate the potential of remote monitoring with digital biomarkers for pediatric clinical trials and care.

\section{Materials and Methods}

This study was performed at the Juliana Children's Hospital (The Hague, the Netherlands) in collaboration with the Centre of Human Drug Research (CHDR) in Leiden between November 2018 and March 2020 and was conducted in compliance with the Good Clinical Practice and the Dutch Code of Conduct regarding medical research with minors. Signed informed consent was obtained from parents or the legal guardian and of children $\geq 12$ years prior to any study-mandated procedure.

\section{Subjects and Study Design}

Patients aged 2-12 years admitted to the pediatric ward of the Juliana Children's Hospital due to CAP $(n=30)$, PW $(n=30)$ and $\mathrm{AE}(n=11)$ were recruited. Patients with a history of chronic illness other than the studied disease were excluded. Patients were enrolled in the study as quickly as possible after admittance and were monitored during hospital admission, a 14-day recovery period immediately after hospital discharge, and another 10-day period 40 days after hospital discharge. The schedule detailing the study periods can be found in Figure 1. Subjects admitted due to nonmedical reasons were excluded from participation. There were no formal discharge criteria. In general, patients were discharged when they were no longer oxygen therapy-dependent during the night. In addition, patients with asthma or PW were discharged when nebulization with bronchodilators was no longer necessary.

\section{Study Assessments}

During the study periods, all patients were asked to continuously wear a Withings ${ }^{\circledR}$ Steel HR Smartwatch (Withings, Issyles-Molineux, France), which collects step count, HR, and sleep pattern. Furthermore, subjects were asked to complete a daily questionnaire. An existing respiratory symptom questionnaire was adapted for a pediatric population, and subjects and their parents of the CAP and PW cohorts were asked to complete the questionnaire at the end of each day to determine a respiratory symptom score (online suppl. Text S1; for all online suppl. material, see www.karger.com/doi/10.1159/000516328) [16]. In addition, these patients performed daily temperature measurements with the Withings Thermo (Withings, Issy-les-Molineux, France). Patients with AE completed the modified Asthma Control Diary (ACD) [17] and spirometry measurements with the Air Next spirometer (NuvoAir, Stockholm, Sweden) every day. The spirometer registers the forced vital capacity, the forced expiratory volume in the first second, and the forced vital capacity/forced expiratory volume ratio [18]. All devices were connected to a G6 smartphone (Motorola, Chicago, IL, USA) with the HealthMate, Thermo, and CHDR MORE ${ }^{\circledR}$ applications preinstalled. The devices used during the study have previously been used in an initial validation study in healthy children [19]. Additionally, the smartphone calendar was filled with a personalized study schedule. At the end of the study, the participants were asked to complete a questionnaire regarding the study experience. Baseline and admission characteristics were obtained from patient charts. 
Fig. 1. Study schedule.

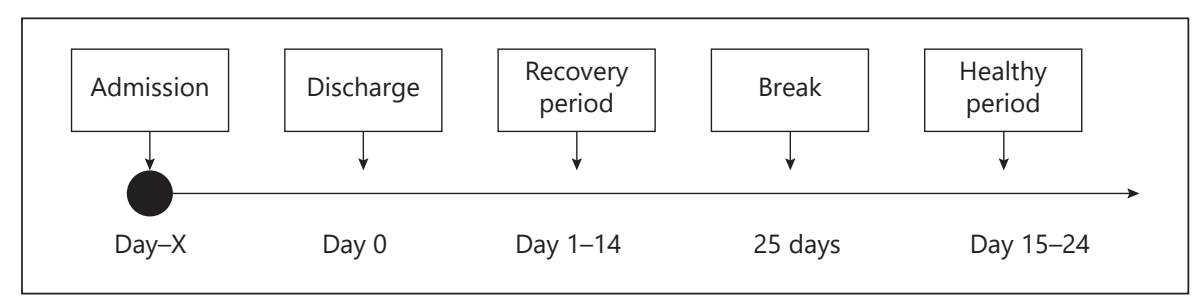

Analysis and Statistics

Compliance and Baseline Characteristics

Compliance was determined by dividing the sum of the completed measurements by the total of the expected measurements for each subject, and the median compliance and interquartile range (IQR) were calculated. Compliance was calculated for the complete study period and for the recovery and healthy period separately. Descriptive statistics were used to describe the baseline and admission characteristics.

\section{Modeling Analysis Set}

The primary endpoint in this study was physical activity (step count). However, individual exploratory plots of physical activity over the entire study period (online suppl. Fig. S2) showed a large amount of interindividual variability between subjects. To define a common point of recovery (return to "healthy" physical activity levels), subjects' data were normalized based on data gathered during the 10-day "healthy" period (days 15-24), since a true baseline period was obviously not possible within the study design. For each individual subject, the mean steps in the healthy period (minimum of 2 days) were used as their "baseline" physical activity (100\%). If no data of the healthy period were available, the mean steps of the last 4 days of the recovery period (minimum of 2 days) were used as reference. Thus, only subjects who performed measurements for at least 2 days in the healthy period or at least 2 days in the last 4 days of the recovery period were included in the analysis set.

\section{Symptom Score Model}

To visualize a recovery trajectory, a descriptive linear mixed model was fitted to model the respiratory symptom score and ACD6 score for the 3 groups separately using the restricted maximum likelihood approach. In this analysis, symptom scores reported before 12:00 p.m. were assumed as data from the previous day. Time was included as a spline covariate with a maximum of 3 degrees of freedom to assess nonlinear recovery. The subject was included as a random intercept. During analysis, contribution to model fit was assessed via a likelihood ratio test and by appraising the Akaike Information Criterion (AIC) and proportion of variance explained $\left(R^{2}\right)$. Model assumptions were checked by inspection of residual plots. Log transformation was performed in the presence of heteroscedasticity. First-order autoregression on the time variable was included to account for temporal autocorrelation.

\section{Physical Activity Model}

Physical activity was modeled descriptively using similar methods. In the model, the study day number was included as a spline covariate with a maximum of 3 degrees of freedom, and a subject was included as a random intercept. The watch wear time between 6:00 a.m. and 10:00 p.m. was included as separate covariate to adjust for partial noncompliance during the day. Estimated mean (95\% confidence interval [CI]) physical activity was calculated over time, with wear time of the watch held constant at $100 \%$. Admission duration, oxygen saturation, respiratory rate, and $\mathrm{HR}$ at admission were included in the models separately as part of an exploratory analysis to assess their effect on recovery.

Heart Rate

Average HR during the day (6:00 a.m.-10:00 p.m.) and average nocturnal HR (12:00 a.m.-5:00 a.m.) were both fitted with a mixed effects model with a subject as a random intercept and time as a spline covariate. Age was included as additional covariate.

Relationship between Recovery Trajectories

In order to quantify the relationship between the 3 estimated recovery trajectories, Pearson correlations were performed to quantify the relationship between estimated mean daily physical activity, HR, and symptom score.

\section{Software}

PySpark version 2.4.6 was used for data aggregation and tabulation. The statistical analyses were performed using $\mathrm{R}$ version 3.6.1 with R-packages nlme, emmeans, and ggeffects.

\section{Results}

\section{Baseline and Admission Characteristics}

Of the 71 patients included in the study, 20 subjects dropped out shortly after inclusion due to discomfort for the child and were excluded during analysis. This majority of dropped out subjects were $2(n=5)$ or $3(n=6)$ years old. The remaining study population consisted of 19 pneumonia patients, 21 PW patients, and 11 asthma patients. Baseline and admission characteristics are shown in Table 1. Of the 51 subjects shown in Table 1, 39 subjects completed measurements for either at least 2 days in the healthy period or at least 2 days in the last 4 days of the recovery period and, thus were suitable for inclusion in the modeling dataset.

\section{Compliance}

For the 51 subjects who completed the study, compliance was determined separately for the entire study period, the recovery period, and healthy period (Fig. 2). Median compliance was 47\% (IQR 33-81\%) during the entire study 


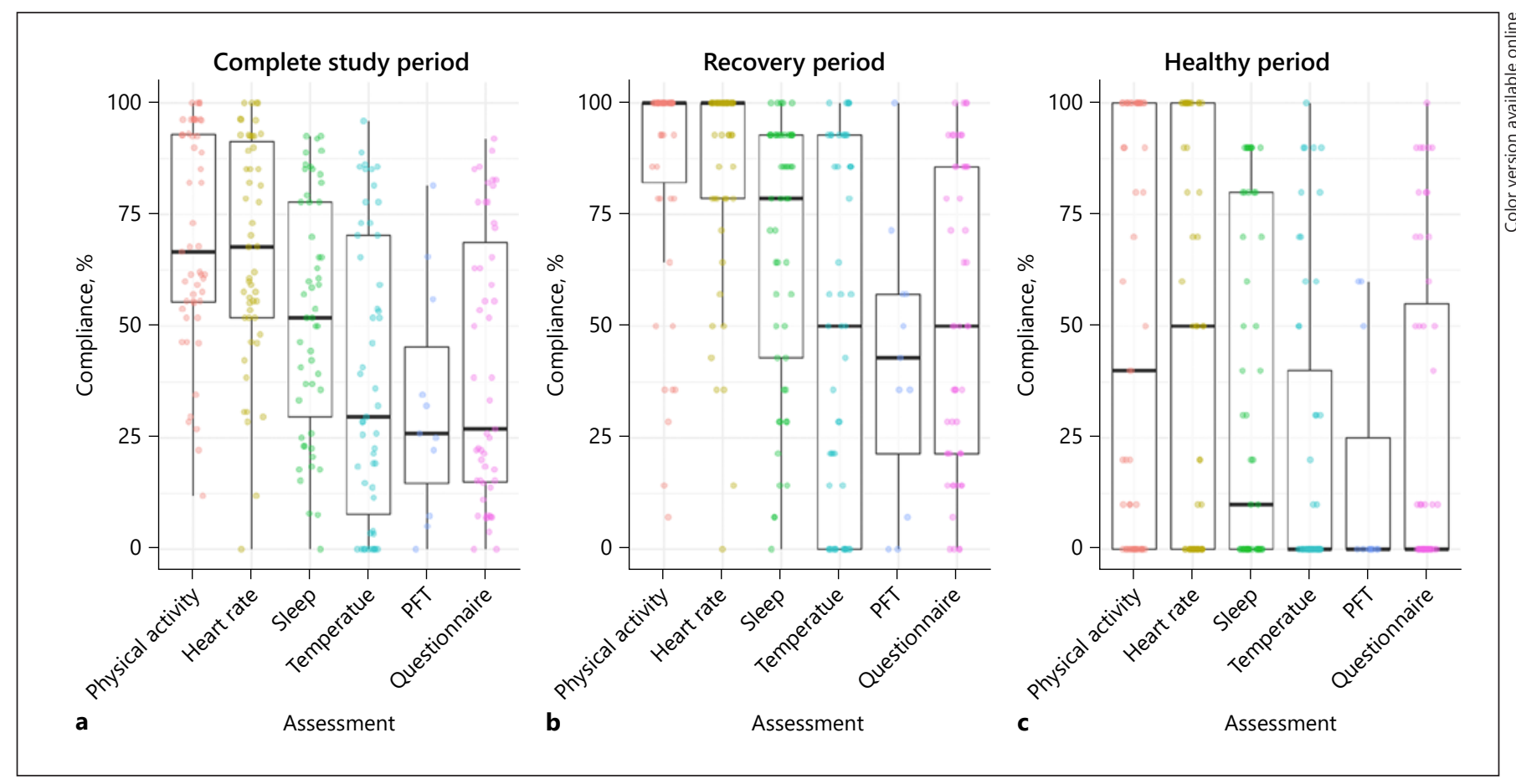

Fig. 2. Compliance to study tasks. Median (IQR) compliance for all measurements combined and for individual measurements. Each dot represents an individual subject. Temperature assessments were performed by CAP and PW subjects, while PFTs were performed by AE subjects. Entire study period (a), 14-day recovery period (b), 10-day healthy period after a 25-day break (c). IQR, interquartile range; CAP, community-acquired pneumonia; PW, preschool wheezing; AE, asthma exacerbation; HR, heart rate.

Table 1. Baseline and admission characteristics

\begin{tabular}{lcccc}
\hline & $\begin{array}{l}\text { All subjects } \\
(n=51)\end{array}$ & $\begin{array}{l}\text { CAP } \\
(n=19)\end{array}$ & $\begin{array}{l}\text { PW } \\
(n=21)\end{array}$ & $\begin{array}{l}\text { AE } \\
(n=11)\end{array}$ \\
\hline Age (mean \pm SD), years & $4.9 \pm 2.9$ & $3.6 \pm 2.0$ & $3.7 \pm 1.2$ & $9.3 \pm 2.2$ \\
Gender, Male \% & 68.6 & 63.2 & 76.2 & 63.6 \\
Ethnicity, Caucasian \% & 45.1 & 52.6 & 38.1 & 45.5 \\
Admission duration (mean \pm SD), h & $62.2 \pm 47.0$ & $63.6 \pm 27.3$ & $48.7 \pm 21.6$ & $85.3 \pm 84.6$ \\
Admission HR (mean \pm SD), bpm & $147.0 \pm 23.3$ & $151.6 \pm 23.9$ & $152.6 \pm 20.1$ & $128.3 \pm 18.6$ \\
Admission respiratory rate, mean breaths/min & $43.1 \pm 15.4$ & $44.8 \pm 13.9$ & $45.1 \pm 16.8$ & $36.7 \pm 14.0$ \\
Admission oxygen saturation (mean \pm SD), $\mathrm{O}_{2} \%$ & $92.8 \pm 3.1$ & $92.2 \pm 3.2$ & $92.6 \pm 2.9$ & $93.6 \pm 3.5$ \\
Oxygen therapy, yes \% & 86.3 & 94.7 & 90.5 & 63.6 \\
Smoking at home, yes \% & 23.5 & 26.3 & 9.5 & 45.5 \\
Family history of respiratory problems, yes \% & 62.7 & 52.6 & 61.9 & 81.8 \\
Day care attendance, yes \% & 23.5 & 31.6 & 28.6 & 0 \\
\hline
\end{tabular}

CAP, community-acquired pneumonia; PW, preschool wheezing; AE, asthma exacerbation; HR, heart rate; bpm, beats per minute; SD, standard deviation.

period, 68\% (IQR 54-91\%) during the recovery period, and $28 \%$ (IQR $0-74 \%$ ) during the healthy period. There was no clear association between age and compliance (online suppl. Fig. S3). Raw data of the subjects remaining in the final dataset is presented in online suppl. Figure S4. Considering the large number of subjects with multiple days of missing data, a mixed effects modeling approach accounting for both missing data from complete study 


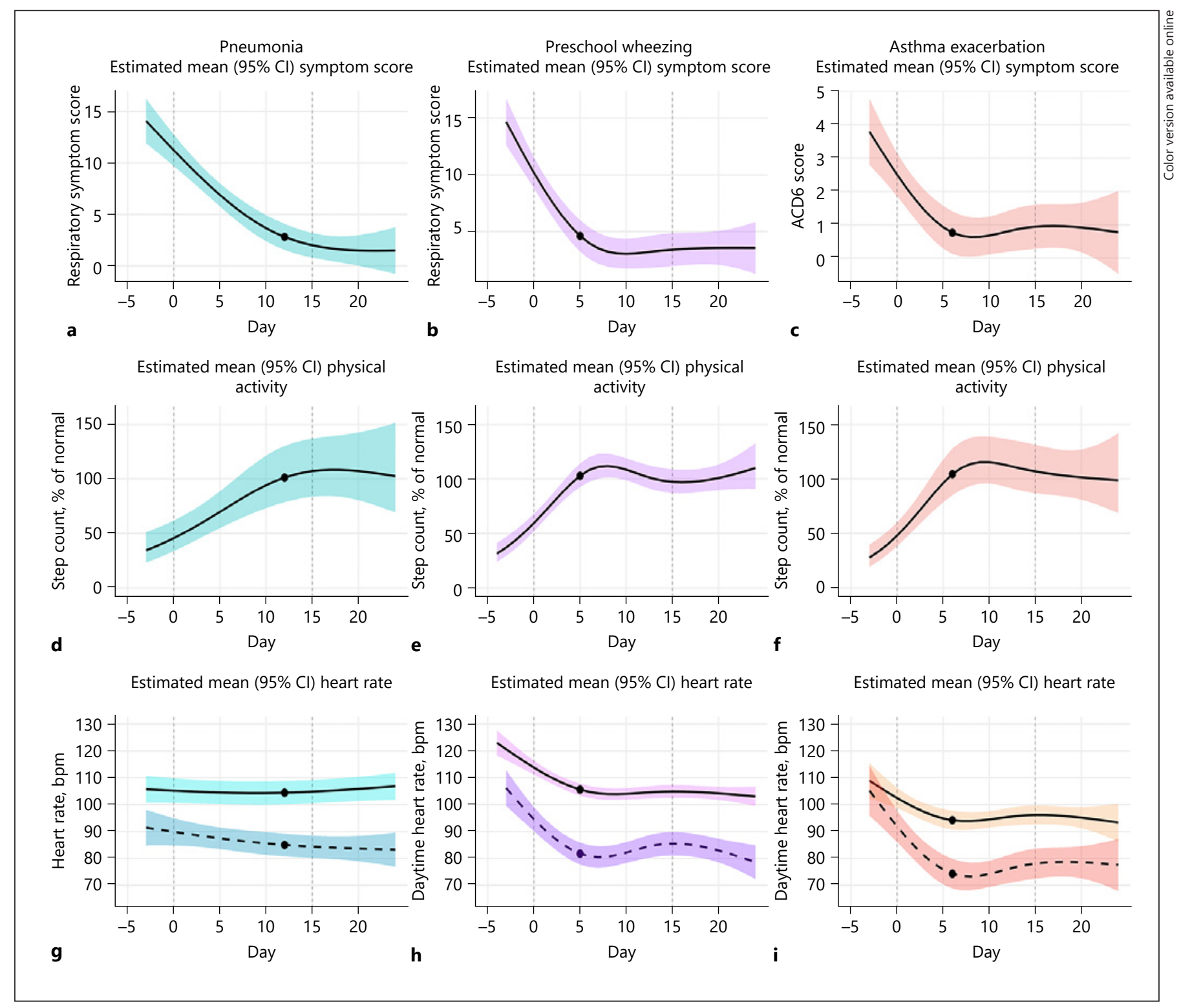

Fig. 3. Estimated recovery trajectory. Estimated mean physical activity over time $(\mathbf{a}-\mathbf{c})$, symptom score over time $(\mathbf{d}-\mathbf{f})$, and daytime and nocturnal HRs ( $\mathbf{g}-\mathbf{i})$ for pneumonia patients (left column), PW patients (central column), and asthma patients (right column). The black lines indicate the estimated population mean; shaded areas represent the estimated $95 \%$ CIs of the mean. The black dots indicate at the day where physical activity first reaches $100 \%$ of normal levels. For HR, darker shaded areas and dotted lines represent nocturnal HR, and lighter shaded area and solid lines represent daytime HR. Estimated $\mathrm{HR}$ was adjusted for age. $\mathrm{HR}$, heart rate; $\mathrm{PW}$, preschool wheezing; $\mathrm{CI}$, confidence interval; $\mathrm{AE}$, asthma exacerbation. days with a random effect structure and for partial noncompliance during the day by adjusting for wear time was most appropriate.

\section{Symptom Score Decreases over Time after Discharge}

ACD6 and respiratory symptom scores were modeled for each diagnosis. Estimated mean symptom scores are displayed in Figure 3a-c. For CAP patients, the average respiratory symptom score decreased from 11.2 (95\% CI 9.7-12.7) at discharge to 2.8 (95\% CI 1.5-4.1) at day 12. PW patients exhibited a mean symptom score of 10.2 (95\% CI 8.8-11.6) at discharge, which decreased towards $4.6(3.2-6.0)$ at day 5 and plateaued after this time point. Finally, subjects with $\mathrm{AE}$ used a different questionnaire 
and exhibited an ACD6 score of 2.5 (95\% CI 1.9-3.1) at discharge and reached a plateau after day 6 (mean 0.8 , $95 \%$ CI 0.1-1.4). Final model coefficients are displayed in online suppl. Table S5.

\section{Physical Activity Displays an Inverse Pattern \\ Compared to Symptom Score}

Estimated mean physical activity for each disease group is displayed in Figure 3d-f. At discharge, physical activity of the CAP group was $46 \%$ of normal levels, and on average, patients achieved $100 \%$ of normal physical activity levels after 12 days. The PW population had a mean physical activity of around 59\% of normal levels at discharge and achieved 100\% physical activity earlier compared to CAP patients after 5 days. The AE population had an estimated mean physical activity of around $48 \%$ at discharge and reached a mean of $100 \%$ after 6 days. Final model coefficients are displayed in online suppl. Table S5.

Admission duration, $\mathrm{O} 2$ saturation, $\mathrm{HR}$, and respiratory rate were separately introduced to the final models as covariates during exploratory analyses. If these variables influence the average recovery trajectory of patients, model fit should improve significantly. However, only admission duration improved model fit in the case of CAP $(\Delta$ AIC $17, p<0.001)$ and PW ( $\Delta$ AIC 9, $p=0.002)$. Other variables did not improve model fit. Estimated model effects for the CAP and PW group are displayed in online suppl. Figure S6.

\section{$H R$ Decreases in $P W$ and $A E$}

Daytime and nocturnal HRs were modeled using the same subjects as for the analysis of physical activity and symptom scores. The mean daytime HR at discharge was $114 \mathrm{bpm}$ for subjects with PW, which decreased to 105 bpm after 5 days. For subjects with asthma, the mean HR during the day was $102 \mathrm{bpm}$ at discharge and stabilized at $94 \mathrm{bpm}$ after 6 days, after which the HR remained at a stable level until the end of the study. For subjects with pneumonia, no such pattern was observed. The nocturnal HR was modeled separately and displayed similar trends. The estimated mean HR over time is displayed in Figure $3 g-i$. Final model coefficients are displayed in online suppl. Table S5.

\section{Estimated Mean Symptom Score, Physical Activity, and Heart Rate Are Correlated}

Correlations between model-predicted mean symptom score, physical activity, and nocturnal HR were performed to quantify the relationships between the estimat- ed recovery trajectories (Fig. 4). Physical activity was inversely related to the symptom score, and HR was positively correlated with the symptom score for all 3 disease groups.

\section{Temperature, Sleep, and Spirometry}

The marginal mean total sleep duration per diagnosis was estimated and is displayed in online suppl. Figure S7. Total compliance for PFTs and temperature was considered too low to attempt further analysis. However, individual plots of a highly adherent subject (online suppl. Fig. S8) showed that PFTs in the home setting have the potential to differentiate between the acute and recovered states in the case of good compliance.

\section{Subject Satisfaction}

Twenty subjects completed the end-of-study questionnaire. The watch scored 2.5 out of 5 on account of being "painful" but also scored 4.0, 3.5, and 4.1 out of 5 for being "fun," "comfortable," and "easy to wear all day," respectively. Five (25\%) parents reported the watch caused some discomfort, itching, irritable skin, or a rash, and $85 \%$ of subjects would be willing to participate in a similar study in the future.

\section{Discussion}

This study is one of the first to describe the at-home recovery trajectory of some of the most common respiratory diseases in pediatrics. The subjects were monitored using an electronic home-monitoring platform including a questionnaire and smartwatch. Home-monitoring with digital devices is often cited as a promising tool for the future, and the noninvasive nature of the measurements may be particularly useful in the field of pediatrics. In this study, we use multiple methods to quantify postdischarge recovery.

The first method, a symptom score questionnaire, is well known and currently the standard in pediatric studies conducted in an at-home setting [20]. On average, children admitted for CAP became symptom-free 5-6 days later compared to $\mathrm{PW}$ and $\mathrm{AE}$ patients. The duration for recovery of $\mathrm{PW}$ and $\mathrm{AE}$ corresponds with findings by Bacharier et al. [4] and Ahmed et al. [21]. A study in 522 children with suspected pneumonia found a median recovery time of 7 days, and two-thirds of children were completely recovered after 12 days [22]. The difference in recovery time between CAP and PW/AE patients is in line with the view that bacterial infections lead to more severe 


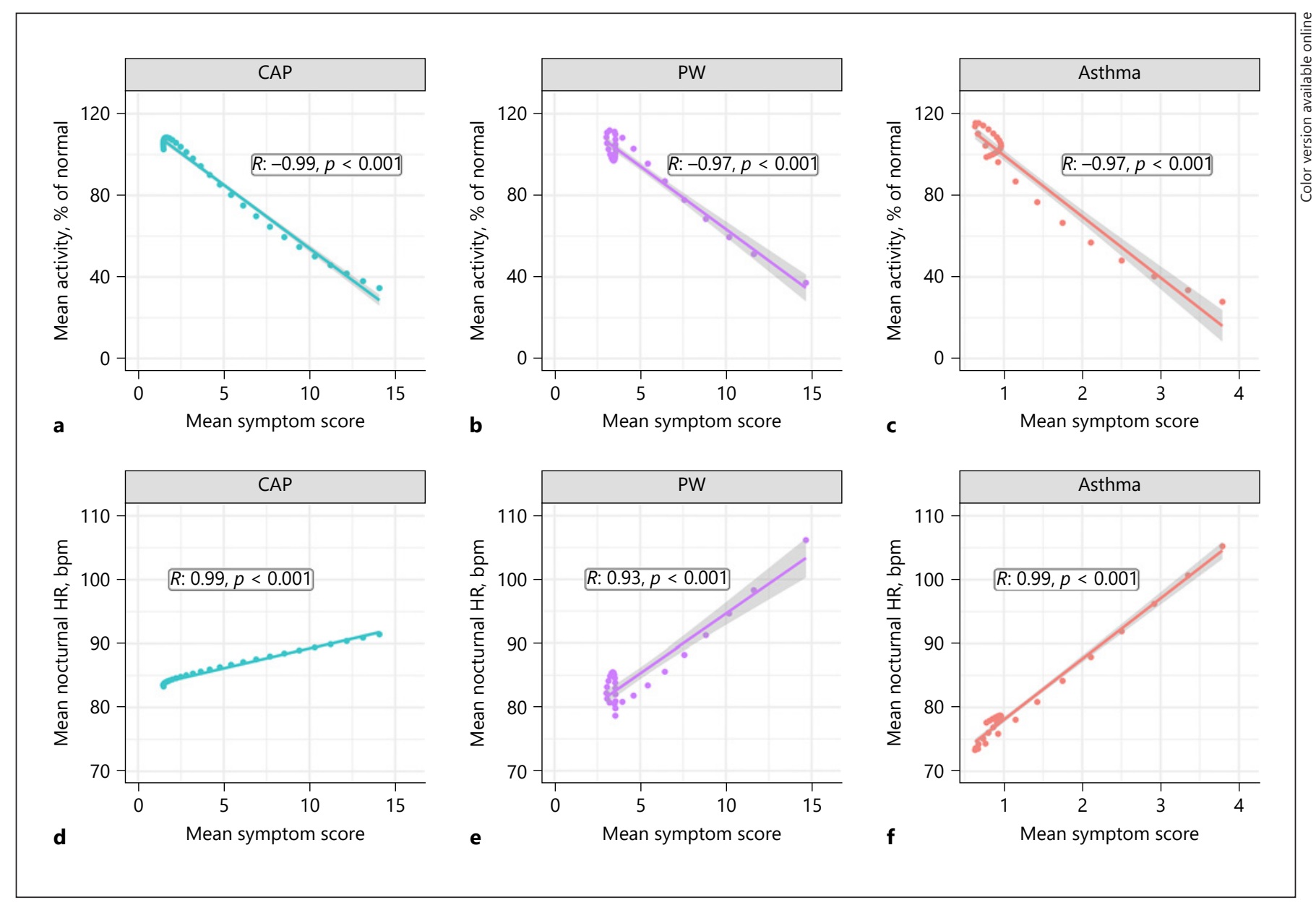

Fig. 4. Correlation between traditional and novel methods to quantify recovery. Pearson correlations between model-estimated mean symptom score and model-estimated mean physical activity (a-c) and between model-estimated mean symptom score and

illness than viral infections. The second method to quantify recovery in this study was to measure physical activity (step count) with a smartwatch. The recovery trajectory was characterized relative to the normal physical activity levels exhibited during the healthy period or final days of the recovery period. The estimated mean physical activity followed an inverse pattern over time compared to the estimated mean symptom score, and the curve reached a plateau at the same time point compared to the modeled symptom score for all 3 patient populations. Third, we assessed daytime and nocturnal HR as a marker for recovery. The estimated mean HR during recovery showed a sharp reduction from admission until around 6 days after discharge for subjects with PW and AE. This corresponded well with the symptom score and physical activity recovery trajectories for both disease groups. nocturnal HR (d-f) for each patient group. HR, heart rate; bpm, beats per minute; CAP, community-acquired pneumonia; PW, preschool wheezing.
However, the HR remained stable throughout the study period for the CAP group. Although the initial reduction in the HR for the subjects with PW and AE may be partly due to reduced respiratory distress, we hypothesize the observed HR decrease is also explained by the frequency of $\beta 2$-agonist administration [23]. Children with PW and AE usually use more bronchodilators at home during the first days after discharge than their regular treatment regimen.

Interestingly, the 3 methods estimated highly similar recovery trajectories, as confirmed by the Pearson correlation coefficients. However, each captures a distinctly different health domain in the form of parental observations, activity behavior, and cardiovascular state. Our data indicate these domains recover at an identical pace, and although future research should confirm these 
observations, the 3 methods may be used interchangeably for this application. In this regard, an advantage of physical activity monitoring is the clear definition of recovery in the form of return to $100 \%$ of normal activity. For HRs and symptom scores, such definitions were not used. Interestingly, we found a strong correlation between mean symptom scores and mean nocturnal HRs for the CAP group, even though the absolute reduction in HRs was much lower than that in the $\mathrm{PW}$ and $\mathrm{AE}$ groups.

A possible application of remote monitoring is the prediction of the length and trajectory of the recovery for individual patients, based on their diagnosis, disease severity, and baseline characteristics. To evaluate whether this is a valid avenue to pursue, we introduced several admission variables as additional covariates in the physical activity models during exploratory analyses. Only the admission duration improved model fit for subjects with CAP and PW. Admission $\mathrm{O}_{2}$ saturation, $\mathrm{HR}$, and respiratory rate as covariates did not improve model fit, and the CI of the estimated means were wide, most likely due to the limited size of the dataset; the directions of the estimated effects for, for example, admission $\mathrm{O}_{2}$ saturation, are plausible and may indicate that, with more data, it is possible to develop a model that is not only able to describe a study population but also estimate individual patients' expected recovery trajectory based on additional clinical characteristics besides diagnosis. Taking this even further, real-time monitoring could detect when children deviate from their expected recovery trajectory and may serve as a warning sign of pending readmission. To realize this option, a larger and, ideally, more complete dataset is needed to adequately isolate the effects of a large number of covariates on the recovery trajectory simultaneously. Furthermore, a system such as this can only add value to standard of care if the data collection and analysis is completely automated and integrated in electronic patient dossiers, and this requires very little input from healthcare providers. If this would be realized, a warning could be sent to caregivers in the case of red flags, prompting reevaluation and modification of the treatment plans to avoid readmission of patients.

Our findings also provide perspective for future pediatric clinical trials. Currently, interventional clinical trials in pediatric patients commonly follow up on predefined time points for objective clinic-based measurements, which are limited to a handful of visits, which provide snapshots of a patient's health status [24, 25]. Another option is to make extensive use of paper questionnaires, which are more subjective $[8,26]$. Remote monitoring platforms could enable researchers to obtain more objective measurements while reducing the necessity of frequent in-hospital follow-up visits. This will automatically lead to a decreased burden for subjects, which may in turn lead to improved recruitment rates, while simultaneously providing a more complete picture of disease activity than traditional trial designs [14].

A major limitation of this study is that significantly less data were collected than originally planned. Of the $71 \mathrm{pa}-$ tients recruited during this study, only 39 subjects were included in the final analysis set. Overall median compliance to study tasks was only $47 \%$, but measurements involving the smartwatch exhibited a higher compliance (67\% for physical activity) than the more traditional symptom questionnaire (27\%), and compliance was higher during the more important recovery period. A number of subjects aged 2 and 3 years dropped out due to smartwatch discomfort, which could relate to the design of the wearable device, which is marketed to adults. A smaller device capable of collecting the same parameters could improve the adherence to study tasks, for example, via a T-shirt or other smart clothing $[27,28]$. The 25 -day break between study periods could have contributed to the low compliance as well. Another limitation is that the respiratory symptom questionnaire used by patients with CAP and PW is not formally validated for use in pediatrics, although no validated questionnaire was available during the conception of this study.

The observed differences in recovery time between groups could not only be explained by the underlying illness, but also due to the limitations described above. However, we expect that the limitations of this pilot study could be largely negated with an improved study and wearable design, and we conclude that a smartwatch is a promising tool for remote monitoring of pediatric patients. We were able to determine the length of postdischarge recovery for 3 common pediatric respiratory diagnoses, which was unclear before this study. A strength of this study is the use of mixed-effects models, which can precisely estimate group means in the presence of missing data points. Future studies may replicate the current findings in a new study with improved design and investigate larger sample sizes, allowing for inclusion of multiple covariates in models to predict individual recovery trajectories. Furthermore, digital endpoints could be included as a secondary outcome in future clinical trials investigating the effect of treatments thought to hasten at-home recovery, such as systemic corticosteroids in the case of PW. 


\section{Conclusion}

Physical activity and HR measured with a smartwatch appears a viable tool for investigating postadmission recovery in children, although the investigated watch was not suitable for children $<4$ years. We believe remote monitoring could significantly benefit observational and interventional pediatric clinical trials and possibly clinical care.

\section{Acknowledgements}

The authors wish to thank the clinical trial assistants, data management officers, all study participants, and their parents for their support and enthusiasm during the conduct of this study.

\section{Statement of Ethics}

This study was approved by the Medical Ethics Committee of Zuidwest Holland.

\section{Conflict of Interest Statement}

There are no conflicts of interest.

\section{Funding Sources}

This study was internally funded by the Centre for Human Drug Research.

\section{Author Contributions}

M.D.K. conducted and designed the study, analyzed the data, and wrote the manuscript. A.M. analyzed the data, recruited patients, and wrote the first draft of the manuscript. A.Z. and D.Z. supported data analysis and reviewed the manuscript. F.E.S., G.J.A.D., M.N., and A.F.C. designed the study and reviewed the manuscript. All authors approved the final manuscript as submitted and agreed to be accountable for all aspects of the work.

\section{Data Availability Statement}

All data presented in this manuscript are available from the corresponding author upon reasonable request.

\section{Trial Registration}

The trial was registered at the Dutch Trial Registry (NTR, Trial NL7610).

\section{References}

1 Williams DJ, Hall M, Shah SS, Parikh K, Tyler A, Neuman MI, et al. Narrow vs broad-spectrum antimicrobial therapy for children hospitalized with pneumonia. Pediatrics. 2013;132(5):e1141.

2 Juvén T, Mertsola J, Waris M, Leinonen M, Ruuskanen O. Clinical response to antibiotic therapy for community-acquired pneumonia. Eur J Pediatr. 2004;163(3):140-4.

3 Tan TQ, Mason EO, Wald ER, Barson WJ, Schutze GE, Bradley JS, et al. Clinical characteristics of children with complicated pneumonia caused by Streptococcus pneumoniae. Pediatrics. 2002;110(1 I):1-6.

4 Ahmed S, Jaleel A, Hameed K, Ahmed F, Danish $\mathrm{H}$, Chugtai A, et al. Serum vitamin D concentration in asthmatic children and its association with recovery time from an asthma exacerbation. J Adv Med Med Res. 2015;10(6):1-10.

5 Wildhaber JH, Sznitman J, Harpes P, Straub D, Möller A, Basek P, et al. Correlation of spirometry and symptom scores in childhood asthma and the usefulness of curvature assessment in expiratory flow-volume curves. Respir Care. 2007;52(12):1744-52.

6 Harris M, Clark J, Coote N, Fletcher P, Harnden A, McKean M, et al. British Thoracic Society guidelines for the management of community acquired pneumonia in children: update 2011. Thorax. 2011;66(Suppl 2):ii1.
7 Ducharme FM, Tse SM, Chauhan B. Diagnosis, management, and prognosis of preschool wheeze. Lancet. 2014;383(9928):1593-604.

8 Mandhane PJ, Paredes Zambrano De Silbernagel P, Nwe Aung Y, Williamson J, Lee BE, Spier S, et al. Treatment of preschool children presenting to the emergency department with wheeze with azithromycin: a placebo-controlled randomized trial. PLoS One. 2017; 12(8): $\mathrm{e} 0182411-15$.

9 Coran P, Goldsack JC, Grandinetti CA, Bakker JP, Bolognese M, Dorsey ER, et al. Advancing the use of mobile technologies in clinical trials: recommendations from the clinical trials transformation initiative. Digit Biomark. 2019;3(3):145-54.

10 Beigelman A, King TS, Mauger D, Zeiger RS, Strunk RC, Kelly HW, et al. Do oral corticosteroids reduce the severity of acute lower respiratory tract illnesses in preschool children with recurrent wheezing? J Allergy Clin Immunol. 2013;131(6):1518.

11 Stickland A, Clayton E, Sankey R, Hill CM. A qualitative study of sleep quality in children and their resident parents when in hospital. Arch Dis Child. 2016;101(6):546-51.

12 Greenberg RG, Corneli A, Bradley J, Farley J, Jafri HS, Lin L, et al. Perceived barriers to pediatrician and family practitioner participation in pediatric clinical trials: findings from the Clinical Trials Transformation Initiative. Contemp Clin Trials Commun. 2017;9: 7-12.

13 Izmailova ES, Wagner JA, Perakslis ED. Wearable devices in clinical trials: hype and hypothesis. Clin Pharmacol Ther. 2018; 104(1):42-52.

14 Kruizinga MD, Stuurman FE, Groeneveld GJ, Cohen AF. The future of clinical trial design: the transition from hard endpoints to valuebased endpoints. Handb Exp Pharmacol. 2019;260:371-97.

15 Kruizinga MD, Stuurman FE, Exadaktylos V, Doll RJ, Stephenson DT, Groeneveld GJ, et al. Development of novel, value-based, digital endpoints for clinical trials: a structured approach toward fit-for-purpose validation. Pharmacol Rev. 2020 Oct;72(4):899-909.

16 El Moussaoui R, Opmeer BC, Bossuyt PMM, Speelman P, De Borgie CAJM, Prins JM. Development and validation of a short questionnaire in community acquired pneumonia. Thorax. 2004;59(7):591-5.

17 Juniper EF, Gruffydd-Jones K, Ward S, Svensson K. Asthma control questionnaire in children: validation, measurement properties, interpretation. Eur Respir J. 2010; 36(6):1410-6. 
18 Kruizinga MD, Essers E, Stuurman FE, Zhuparris A, van Eik N, Janssens HM, et al. Technical validity and usability of a novel smartphoneconnected spirometry device for pediatric patients with asthma and cystic fibrosis. Pediatr Pulmonol. 2020 Sep;55(6):2463-70.

19 Kruizinga MD, Heide NV, Moll A, Zhuparris A, Yavuz Y, Kam ML, et al. Towards remote monitoring in pediatric care and clinical trialstolerability, repeatability and reference values of candidate digital endpoints derived from physical activity, heart rate and sleep in healthy children. PLoS One. 2021;16(1):e0244877.

20 Coons SJ, Eremenco S, Lundy JJ, O'Donohoe P, O'Gorman H, Malizia W. Capturing patientreported outcome (PRO) data electronically: the past, present, and promise of ePRO measurement in clinical trials. Patient. 2015;8(4): 301-9.
21 Bacharier LB, Phillips BR, Zeiger RS, Szefler SJ, Martinez FD, Lemanske RF, et al. Episodic use of an inhaled corticosteroid or leukotriene receptor antagonist in preschool children with moderate-to-severe intermittent wheezing. J Allergy Clin Immunol. 2008;122(6):1127-e8.

22 Örtqvist Å, Hedlund J, Burman LÅ, Elbel E, Höfer MA, Leinonen M, et al. Randomised controlled trial of clinical outcome after chest radiograph in ambulatory acute lower-respiratory infection in children. Lancet. 1998; 351(9100):404-8.

23 Sears MR. Adverse effects of beta-agonists. J Allergy Clin Immunol. 2002;110(6 Suppl):S322-8.

24 Esposito S, Tagliabue C, Picciolli I, Semino M, Sabatini C, Consolo S, et al. Procalcitonin measurements for guiding antibiotic treatment in pediatric pneumonia. Respir Med. 2011;105(12):1939-45.
25 Dimopoulos G, Matthaiou DK, Karageorgopoulos DE, Grammatikos AP, Athanassa Z, Falagas ME. Short- versus long-course antibacterial therapy for community-acquired pneumonia: a meta-analysis. Drugs. 2008; 68(13):1841-54.

26 Papi A, Nicolini G, Baraldi E, Boner AL, Cutrera R, Rossi GA, et al. Regular vs prn nebulized treatment in wheeze preschool children. Allergy. 2009;64(10):1463-71.

27 Montoye AHK, Mitrzyk JR, Molesky MJ. Comparative accuracy of a wrist-worn activity tracker and a smart shirt for physical activity assessment. Meas Phys Educ Exerc Sci. 2017;21(4):201-11.

28 Ajami S, Teimouri F. Features and application of wearable biosensors in medical care. J Res Med Sci. 2015 Dec;20(12): 1208-15. 